\title{
AGE-RELATED PRESENCE OF SELECTED VIRAL AND BACTERIAL PATHOGENS IN PARAFFIN-EMBEDDED LUNG SAMPLES OF DOGS WITH PNEUMONIA
}

\author{
Daniela WÖHRER ${ }^{1}$, Joachim SPERGSER ${ }^{2}$, Gabriela BAGRINOVSCHI ${ }^{3}$, Karin MÖSTL $^{3}$ \\ and Herbert WEISSENBÖCK ${ }^{*}$ \\ ${ }^{1}$ Institute of Pathology and Forensic Veterinary Medicine, ${ }^{2}$ Institute of Microbiology, \\ ${ }^{3}$ Institute of Virology, Department of Pathobiology, University of Veterinary Medicine \\ Vienna, Veterinärplatz 1, A-1210 Vienna, Austria
}

(Received 16 July 2015; accepted 28 October 2015)

The aim of this retrospective study was to detect selected pathogens in pneumonic lung tissue of dogs of different age groups by immunohistochemistry (IHC), in situ hybridisation (ISH) or polymerase chain reaction (PCR) in order to get information about their involvement in pneumonia formation. In archived formalin-fixed and paraffin wax-embedded lung samples from 68 cases with the clinical and histologic diagnosis of pneumonia the histological pattern of pneumonia was re-evaluated and the samples were further investigated for the following infectious agents: canine distemper virus (CDV), canine adenovirus type 2 (CAV-2), canine respiratory coronavirus (CRCoV), Bordetella (B.) bronchiseptica, Pasteurella (P.) multocida, Mycoplasma spp., and Pneumocystis spp. In 47.1\% of the samples at least one of the featured respiratory pathogens was detected. In $31.3 \%$ of these positive samples more than one pathogen could be found. The correct detection of CDV had been achieved in ten out of eleven positive cases $(90.9 \%)$ upon initial investigation, but the presence of bacterial pathogens, like B. bronchiseptica (10 cases) and P. multocida (17 cases) had been missed in all but one case. While CDV and CRCoV infections were exclusively found in dogs younger than one year, the vast majority of infections with $P$. multocida and B. bronchiseptica were both common either in dogs younger than 4 months or older than one year. Thus, this retrospective approach yielded valuable data on the presence, absence and prevalence of certain respiratory pathogens in dogs with pneumonia.

Key words: Dog, pneumonia, respiratory disease, respiratory pathogens, retrospective study

In dogs, like in any other domestic animal species, pneumonias belong to the most important infectious diseases. From the morphological point of view, the major inflammation patterns are interstitial pneumonia, bronchointerstitial pneumonia and bronchopneumonia (Caswell and Williams, 2007). The disease

*Corresponding author; E-mail: herbert.weissenboeck@vetmeduni.ac.at;

Phone: 00431 (250) 772-418; Fax: 00431 (250) 772-490 
can take an acute, subacute or chronic course. In younger dogs viral infection followed by bacterial invasion is common, whereas aspiration pneumonia and foreign body pneumonia are more frequently seen in older dogs (Dear, 2014).

Pneumonias of dogs can be caused by a large variety of pathogens including viruses, bacteria, fungi, and parasites. Some pneumonias are initiated by a single pathogen (e.g. canine distemper virus [CDV]), whereas others are due to mixed infections with pathogens of different virulence as in canine infectious respiratory disease (CIRD) also known as the 'kennel cough' complex (Ford, 2012). For many years, CIRD was considered a common disease of limited clinical significance, largely controlled by routine vaccination. Over the last decade there has been a resurgence of interest in canine respiratory pathogens, which has been triggered by the ineffectiveness of vaccines at controlling respiratory disease outbreaks, especially within large dog populations like in kennels or animal shelters. Furthermore, infections with novel pathogens causing more severe clinical signs or fatalities have contributed to the impact of CIRD (Priestnall et al., 2014). CIRD is characterised by infections with viruses, mycoplasmas or Bordetella (B.) bronchiseptica which cause damage to the mucociliary clearance mechanism of the upper airways as well as primary lesions in the lung tissue. In addition, some pathogens, such as canine respiratory coronavirus $(\mathrm{CRCoV})$, are suspected to interfere with the host's immune system and predispose the host to secondary bacterial colonisation and infection (Caswell and Williams, 2007; Ford, 2012; Priestnall et al., 2014).

In the context of this increased relevance of canine respiratory diseases the major aim of the present retrospective investigation was the direct detection of pathogens in pneumonic lung tissue by immunohistochemistry (IHC) or in situ hybridisation (ISH), and, in the case of one pathogen, quantitative reverse transcriptase-polymerase chain reaction (qRT-PCR). This was done with the intention to evaluate whether initially performed routine diagnostics had yielded comparable results and to assess whether infectious agents not tested routinely had escaped attention on a larger scale. The pathogens selected to address the first aim were CDV and the bacteria Pasteurella (P.) multocida and B. bronchiseptica. Pathogens initially not routinely tested for were canine adenovirus type 2 (CAV-2), CRCoV, Mycoplasma spp., and Pneumocystis spp.

\section{Materials and methods}

\section{Tissue samples}

Sixty-eight paraffin wax-embedded lung samples from the archive of the Institute of Pathology and Forensic Veterinary Medicine of the University of Veterinary Medicine Vienna were used. The samples were obtained from dogs which had been necropsied between 1997 and 2007 and in which pneumonia had 
been diagnosed histologically. Data concerning signalment (breed, sex, age), clinical symptoms, results of postmortem examination as well as recorded laboratory findings from initial bacteriological and parasitological examinations were collected. Forty-four of the investigated dogs belonged to 27 different pure breeds, 21 were different crossbreeds, and in three cases the breed was not recorded or unknown. No breed was overrepresented. Twenty-nine dogs were males, two dogs were neutered males, 28 dogs were females and six were neutered females. In three cases the sex was not recorded. The dogs were assigned to the following age groups: $\leq 2$ weeks of age (8), 2 weeks -3 months (22), 4-12 months (13), 1-10 years (19) and $>10$ years (5). In one case the age was not recorded. Six dogs had a history of being kept in an animal shelter.

All lung samples were stained with haematoxylin and eosin (HE) according to standard techniques, and lung lesions were classified regarding type, severity and distribution. Furthermore, the samples were subjected to IHC (CDV, P. multocida, B. bronchiseptica, Mycoplasma spp.) and ISH [CAV-2, Pneumocystis (P.) carinii]. In the case of Mycoplasma, polymerase chain reaction (PCR) was used for the confirmation of IHC results. A qRT-PCR procedure was used for the detection of CRCoV. All methods were performed including positive controls; the PCRs were carried out using negative controls additionally.

\section{Immunohistochemistry (IHC)}

The primary antibodies used, together with their source and dilutions, are listed in Table 1. In the case of Mycoplasma, a mixture of three different antibodies (directed against the canine representatives of the Mycoplasma synoviae cluster: M. cynos, M. canis, and M. edwardii) (Chalker and Brownlie, 2004) was used for initial screening, in order to pick up the most relevant mycoplasmas in the canine respiratory tract in a single assay. Then the positive cases were examined with the antibodies against M. cynos, M. canis, and M. edwardii separately. IHC was performed by the use of an automated immunostainer (Thermo Autostainer 360-2D System, Thermo Fisher Scientific, Fremont, CA, USA) using the Ultravision LP Detection System (Thermo Fisher). Antigen retrieval was achieved with the PT Module system (Thermo Fisher) using citrate buffer ( $\mathrm{pH}$ 6.0).

In situ hybridization (ISH)

This technique was used for the detection of CAV-2 and Pneumocystis spp. The procedures followed previously described protocols (CAV-2: Benetka et al., 2006; Pneumocystis spp.: Binanti et al., 2014). The probe sequences are listed in Table 2.

\section{Polymerase chain reaction}

Five $10-\mu \mathrm{m}$ sections of paraffin-embedded lung tissue were deparaffinised and DNA as well as RNA were extracted using the ALLPrep DNA/RNA FFPE (Qiagen, Vienna, Austria) according to the manufacturer's instructions. 
Table 1

Antibodies used for immunohistochemistry, their source and working dilution

\begin{tabular}{llll}
\hline Pathogen & Antibody type; host species & \multicolumn{1}{c}{ Source } & Dilution \\
\hline Canine distemper virus & Monoclonal, mouse & C. Örvell, Stockholm, Sweden & $1: 30,000$ \\
Bordetella bronchiseptica & Polyclonal, rabbit & Inst. Microb., Vetmeduni $^{*}$ & $1: 10,000$ \\
Pasteurella multocida & Polyclonal, rabbit & Inst. Microb., Vetmeduni $^{*}$ & $1: 40,000$ \\
Mycoplasma canis & Polyclonal, rabbit & Inst. Microb., Vetmeduni $^{*}$ & $1: 40,000$ \\
Mycoplasma cynos & Polyclonal, rabbit & Inst. Microb., Vetmeduni $^{*}$ & $1: 10,000$ \\
Mycoplasma edwardii & Polyclonal, rabbit & Inst. Microb., Vetmeduni & $1: 60,000$ \\
\hline
\end{tabular}

*Institute of Microbiology, University of Veterinary Medicine Vienna, Austria

For the identification of canine mycoplasmas (M. canis, M. cynos and M. edwardii), single species-specific PCRs were performed as described elsewhere (Chalker et al., 2004). Primer sequences and lengths of amplification products are listed in Table 2. The amplification products were visualised by gel electrophoresis and ethidium bromide staining.

For assessment of the presence of $\mathrm{CRCoV}$ a previously described qRTPCR was applied (Spiss et al., 2012). The primers used, the probe sequences and the length of the amplification products are listed in Table 2.

\section{Results}

\section{Clinical signs and postmortem examination}

Thirty-nine (57.4\%) of the investigated dogs had respiratory problems like gasping of breath, dyspnoea and clinical signs of rhinitis, conjunctivitis, tracheitis, bronchitis, and pneumonia. In seven cases $(10.2 \%)$ neurological signs and in 12 cases $(17.6 \%)$ gastrointestinal signs were noticed. Twenty-six $(38.2 \%)$ of the dogs had died and five (7.4\%) (affected dogs or their mothers) were verifiably not vaccinated. At postmortem examination all 68 cases showed a form of pneumonia, and in a number of cases additional relevant diagnoses had been recorded as well (16: bronchitis, 3 : pleuritis, 30: enteritis, 8 : encephalitis, 24: splenomegaly).

\section{Histopathological findings}

The histological pattern of pneumonia was re-evaluated in all cases. Sometimes there were different types of pneumonias in different lung areas. Classification and assignments to groups were based on the most widespread type of inflammation.

In 14 cases the lesions were present in all lung lobes. In 13 cases the caudal lobes and in 11 cases the cranial and medial lobes were most severely affected. In 30 cases the macroscopic localisation of the inflammation was not recorded. 
VIRAL AND BACTERIAL PATHOGENS IN DOGS WITH PNEUMONIA

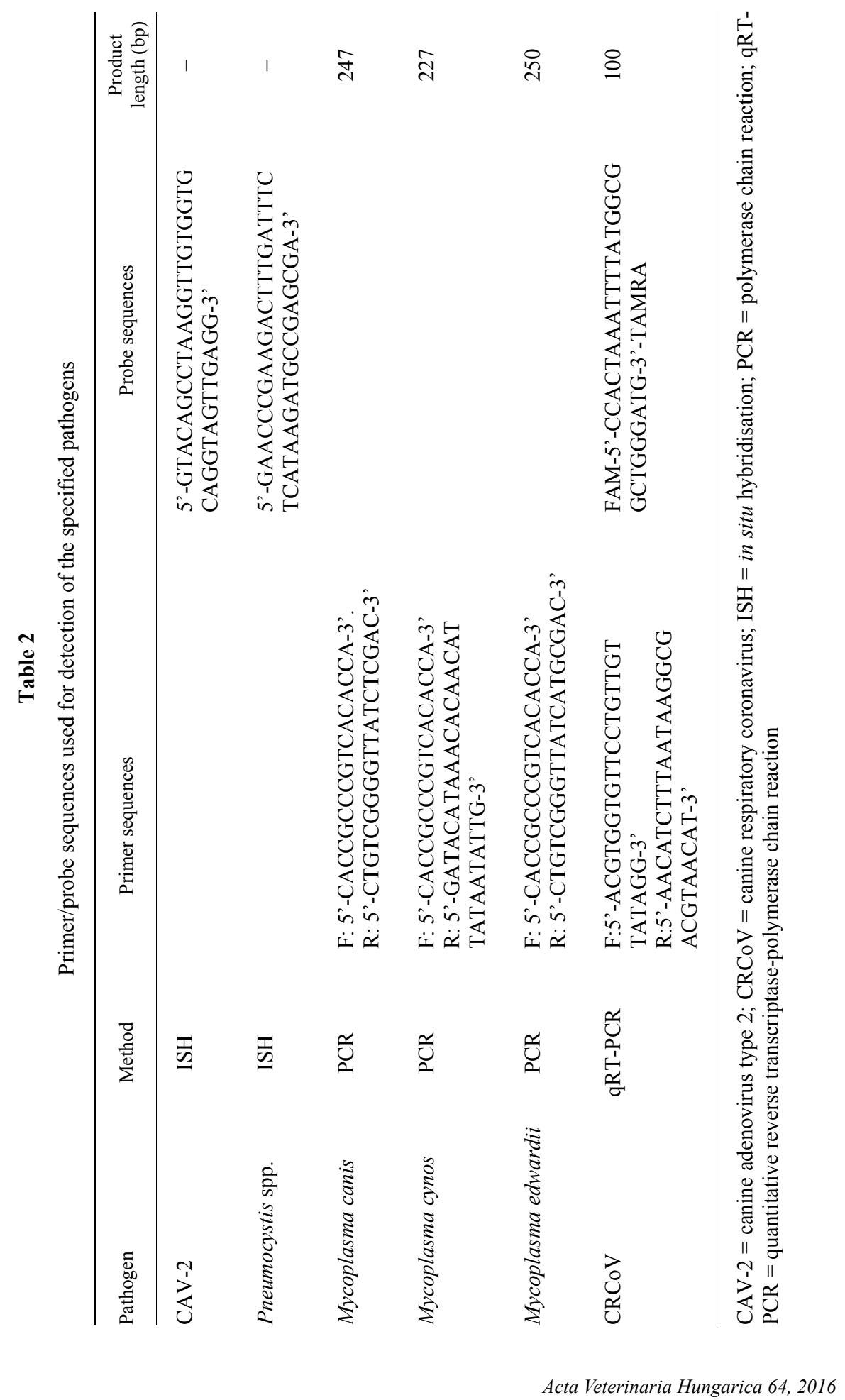




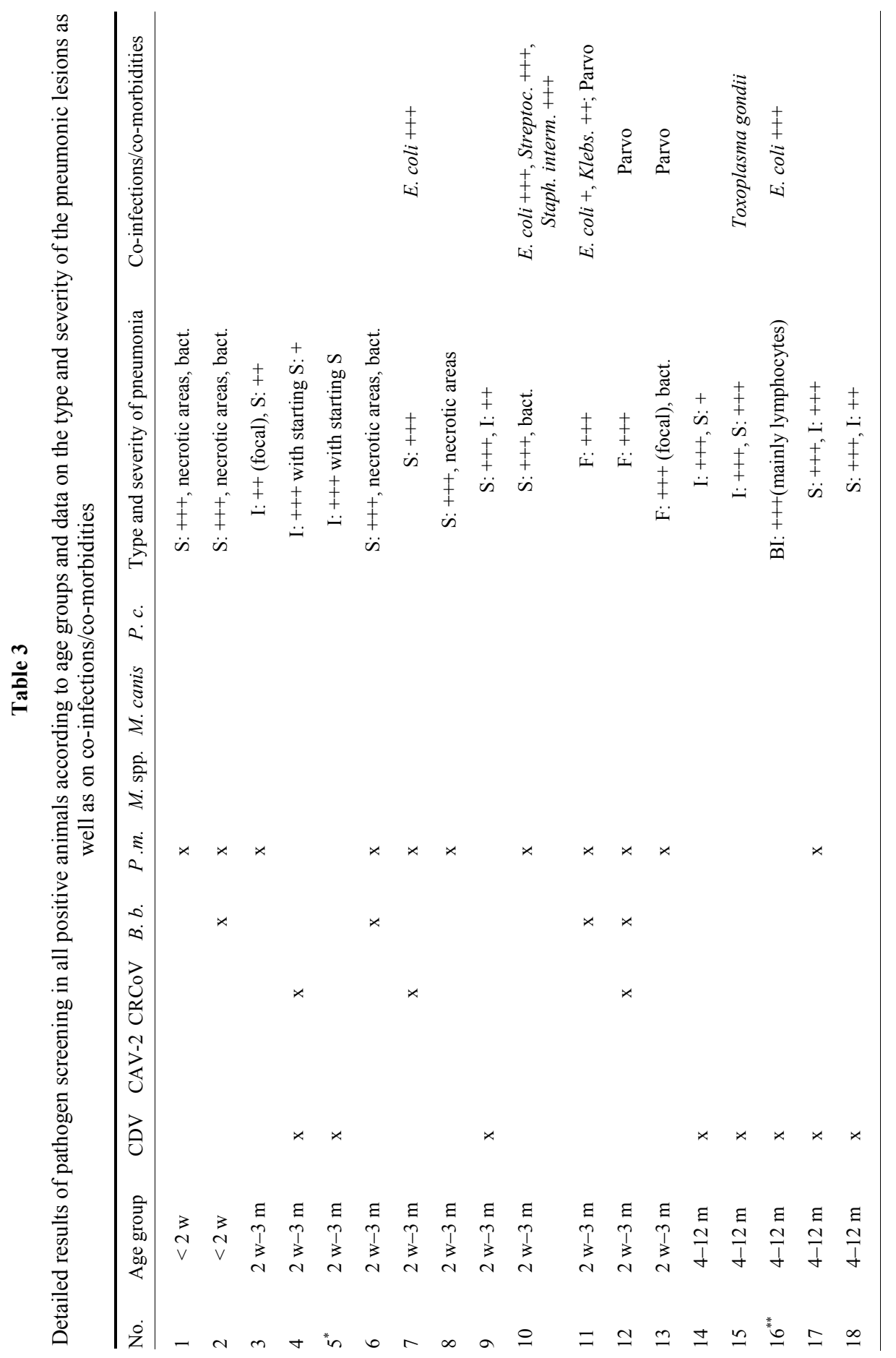




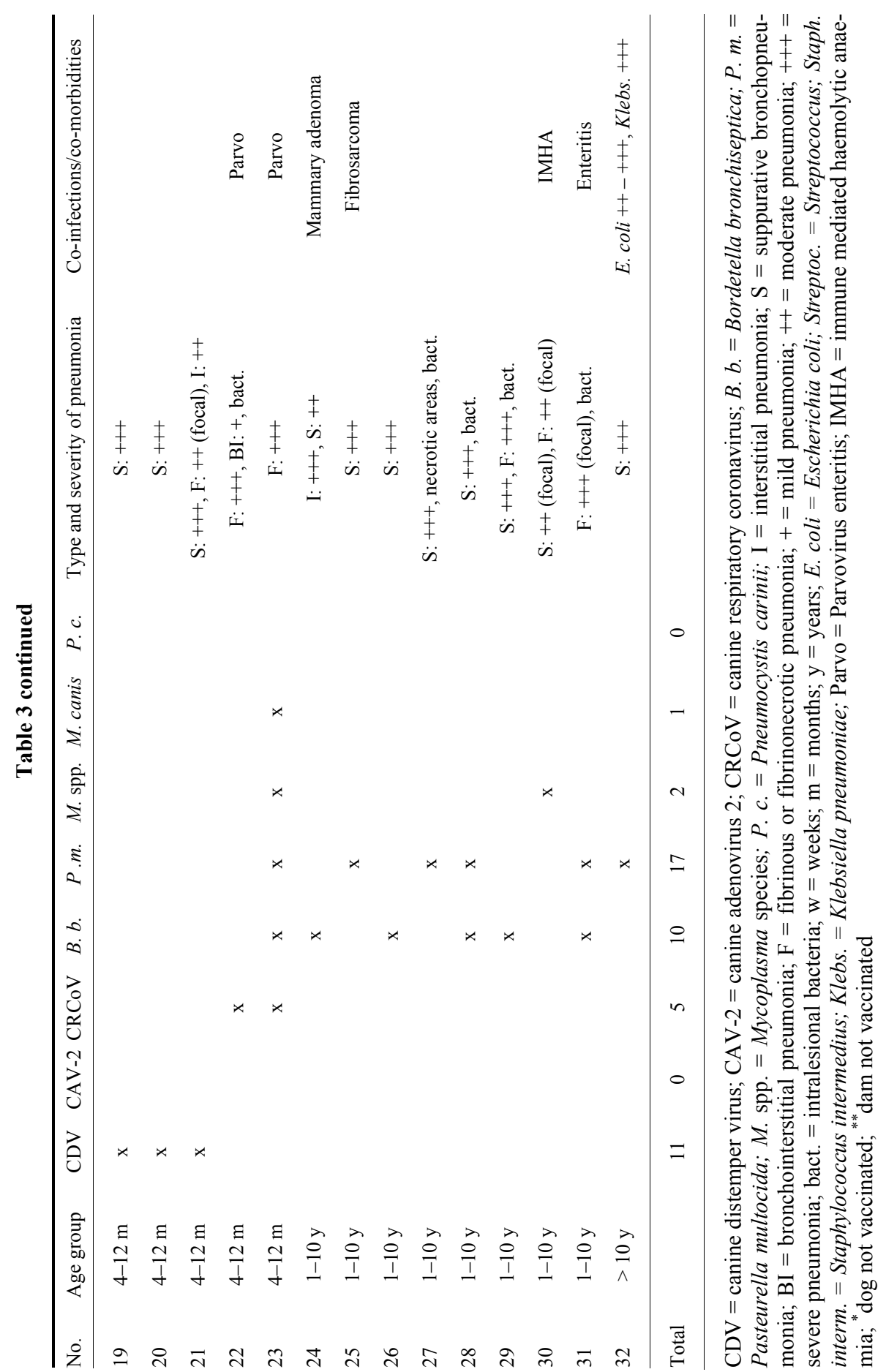


Histologically, there were variable grades of suppurative bronchopneumonia in 42 animals. In six of these cases there were additional widespread necrotic areas. This type of inflammation was characterised by purulent exudate in bronchi, bronchioles and alveoli, which consisted mainly of neutrophil granulocytes, exfoliated epithelial cells and alveolar macrophages. Nineteen of these 42 cases showed an additional interstitial pneumonia, which was characterised by pneumocyte type II hyperplasia and the presence of lymphocytes, macrophages and plasma cells in the intralobular interstitium.

In the remaining 26 cases, a fibrinonecrotic pneumonia, sometimes with a more or less prominent purulent component was found. The inflammation of the lung tissue was accompanied by severe hyperaemia in 24 cases, thrombosis of arterial blood vessels in 15 cases, and variable amounts of intralesional bacterial colonies in 10 cases as well as intraalveolar extravasates in three cases. Fungal elements were not histologically detected in any case.

\section{IHC, ISH and PCR}

Thirty-two cases $(47.1 \%)$ were positive for at least one of the pathogens investigated. In $10(31.3 \%)$ of these 32 cases more than one pathogen under investigation could be detected in the lung tissue (Table 3 ). In 36 animals none of the featured pathogens was found (Table 4).

In 11 cases $(16.1 \%)$ antigens of CDV could be detected by immunohistochemistry. These were all juvenile dogs (3-9 months of age). The strongest IHC signals were found in the alveolar lumina and walls, weaker signals were present in the perivascular connective tissue and in the bronchi and bronchioles. The positive cells were alveolar, bronchial and bronchiolar epithelial cells, alveolar macrophages, syncytial cells, and not further identifiable interstitial cells (Fig. 1A). Ten of these cases had already been diagnosed upon routine diagnostics, but one additional positive result could be established in the present study. In three cases co-infections (Toxoplasma gondii, P. multocida, $\mathrm{CRCoV}$ ) could be identified.

In 10 cases (14.7\%) antigens of $B$. bronchiseptica could be labelled in intraalveolar bacterial colonies (Fig. 1B) and intracellularly within macrophages and neutrophils. Five of these dogs were adults with ages between one to ten years and five were puppies with ages between one and three months. In only one of these cases had a bacteriological examination of the lungs been performed upon necropsy. Bordetella bronchiseptica was not detected in this case.

In 17 cases $(25.0 \%)$ antigens of P. multocida could be found by IHC (Fig. 1C). Twelve animals were very young puppies with ages ranging from one week to four months, and five animals were adults aged between four and eleven years. The distribution of the signals was comparable to the distribution of Bordetella antigens. In only one out of the five cases which were examined bacteriologically upon necropsy had $P$. multocida been initially diagnosed. 


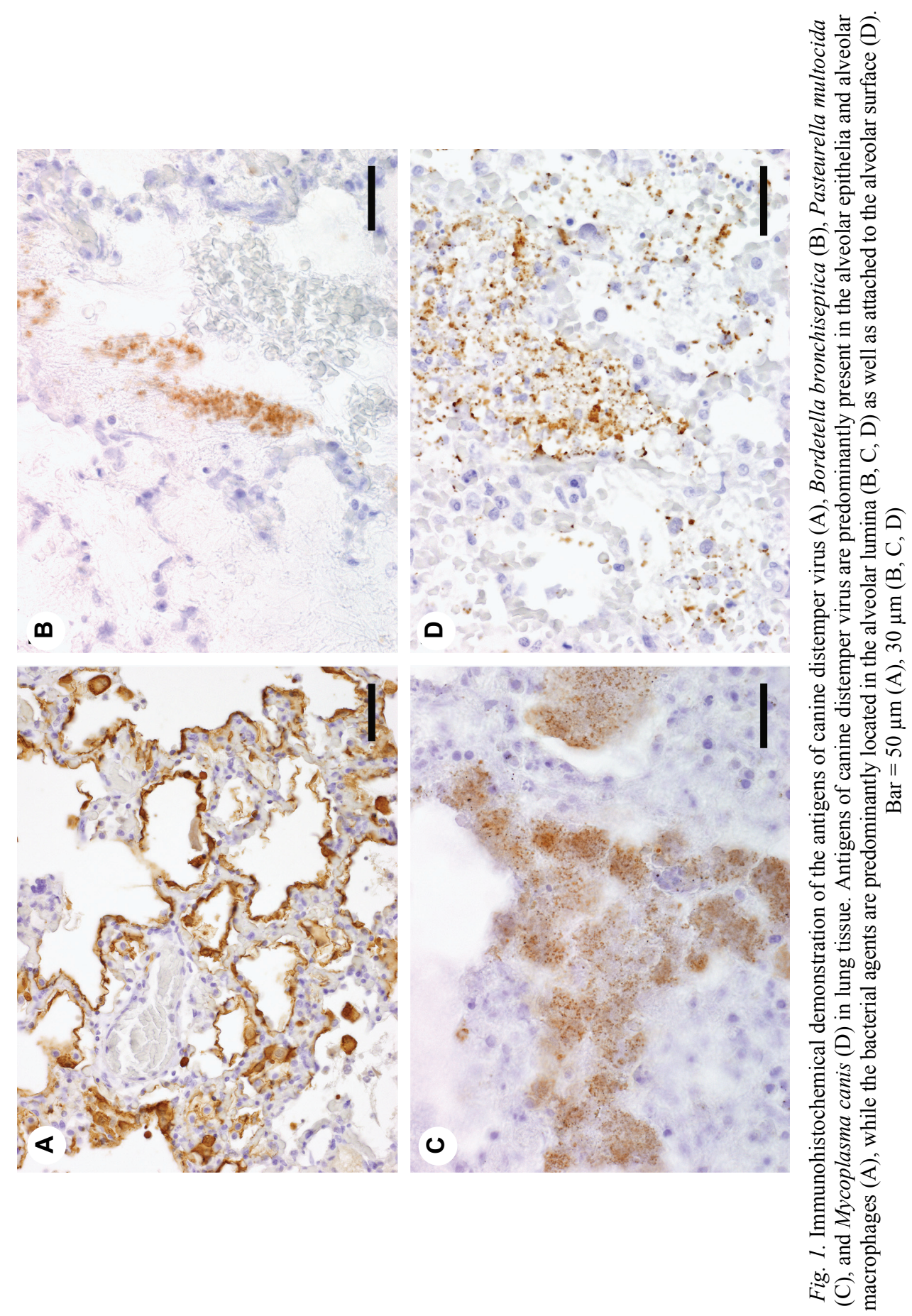


There were seven cases of mixed infections with $B$. bronchiseptica and $P$. multocida. In one of the $P$. multocida cases and in one of the mixed infections (B. bronchiseptica and P. multocida) a co-infection with CRCoV was detected.

Two cases were positive with the anti-Mycoplasma antibody mixture and remained positive in the subsequent IHCs with antibodies for both M. canis and M. edwardii (Fig. 1D). The IHC signal was found in bacterial colonies within the alveolar lumina and attached to the alveolar walls as well as in the cytoplasm of macrophages. In the subsequent PCR examination only one case yielded a positive result (M. canis). The PCR-positive case was found to have a number of coinfections (CRCoV, B. bronchiseptica, P. multocida, and CPV-2).

Table 4

The remaining 36 animals age grouped according to their main type of pneumonia and possible immunocompromising factors

\begin{tabular}{lccc}
\hline Age groups & Number & Type of pneumonia & Identified infections/co-morbidities \\
\hline Under 2 weeks $^{* *}$ & 6 & $\mathrm{~S}(4), \mathrm{F}(2)$ & E. coli (4) \\
2 weeks-3 months & 11 & $\mathrm{~S}(6), \mathrm{F}(5)$ & E. coli (2), Erysipelothrix rhusiopathiae (1) \\
4-12 months & 3 & $\mathrm{~F} \mathrm{(3)}$ & Parvo (1), E. coli (1) \\
1-10 years & 11 & $\mathrm{~S} \mathrm{(4),} \mathrm{F} \mathrm{(7)}$ & $\begin{array}{c}\text { E. coli (1), Septicaemia (1), } \\
\text { Mammary adenoma (2), IMHA (1) }\end{array}$ \\
Above 10 years & 4 & $\mathrm{~S} \mathrm{(3),} \mathrm{F} \mathrm{(1)}$ & \\
n/a & 1 & $\mathrm{~S} \mathrm{(1)}$ & \\
\hline
\end{tabular}

$\mathrm{S}=$ suppurative bronchopneumonia; $\mathrm{F}=$ fibrinous or fibrinonecrotic pneumonia; $E$. coli $=$ Escherichia coli; Parvo = Parvovirus enteritis; IMHA = immune-mediated haemolytic anaemia; "two dogs of this group not vaccinated; ${ }^{* *}$ one dam of this group not vaccinated

In five cases recognisable amplification of $\mathrm{CRCoV}$ was generated by qRTPCR. In one case the Cq value (27.14) was compatible with a high viral load. The four other cases had very high Cq values ( $>37)$.

All 68 lungs examined by ISH for the presence of CAV-2 and for the presence of Pneumocystis spp. were negative.

\section{Discussion}

In this retrospective investigation of paraffin-embedded canine lung samples representing different types of pneumonia, at least one of the featured respiratory pathogens was detected in $47.1 \%$ of the samples. Mixed infections with two or more infectious agents were common (31.3\% of positive cases). CDV was readily identified by IHC in 11 cases. In all except one of the cases this highly contagious pathogen had been correctly determined upon initial diagnostic ex- 
amination. However, one additional case of canine distemper had been identified in the present study which, due to the lack of clinical and histological suspicion, had escaped correct diagnosis. In the canine distemper cases secondary infections with the pathogens investigated here occurred only on a very small scale, an observation which does not support the notion of frequent superinfections in CDV pneumonia (Chvala et al., 2007).

In three dogs a single infection with $B$. bronchiseptica was present, in seven cases $B$. bronchiseptica was accompanied by $P$. multocida and in 10 cases a single infection with $P$. multocida was diagnosed. Bordetella bronchiseptica is regarded one of the principal causative agents of CIRD and may be a critical complicating factor in dogs simultaneously infected with a viral pathogen (Ford, 2012). Interestingly, single infections with $B$. bronchiseptica were only found in adult dogs, while mixed and single infections with $P$. multocida occurred in puppies, juveniles and adults. Pasteurella spp. are members of the normal microflora of the nasopharynx and large airways of dogs. Concurrent viral infections and other stresses or immunosuppressive conditions lead to their proliferation and gaining access to the lower airways. Bacterial proliferation results in an influx of inflammatory cells and cytokine mediators, resulting in fibrinopurulent exudation which is typical of Pasteurella pneumonia (Lee-Fowler and Reinero, 2012). From the 20 cases which had been immunohistochemically diagnosed as single or mixed infections with $B$. bronchiseptica or P. multocida only six had been examined bacteriologically. Only in one case had $P$. multocida been isolated. This discrepancy remains unexplained but could probably have been influenced by antibiotic treatment attempts. Further, the presence of high numbers of bacterial colonies associated with lesions such as necroses and inflammation supports the notion that these bacteria were causally involved in the initiation or progression of the observed lesions and were not just insignificant bystanders or commensals.

In dogs, mycoplasmas are thought to be part of the normal bacterial flora in the upper respiratory tract, but there are inconsistent reports about the presence of mycoplasmas in the lower respiratory tract of healthy dogs (Chalker et al., 2004). There is evidence that M. cynos could be a primary pathogen of CIRD (Priestnall et al., 2014). However, in the current study, using an IHC procedure which had successfully labelled M. cynos previously (Chvala et al., 2007) there was not a single case of $M$. cynos infection and only two cases in which mycoplasmas were identified. By IHC there was a positive reaction with antibodies to M. canis and M. edwardii. As these two Mycoplasma species are closely related phylogenetically (Chalker and Brownlie, 2004), this most likely represents a cross-reaction of the antibodies used. PCR for Mycoplasma species discrimination was positive in only one case, confirming $M$. canis as the involved species. The negative result of PCR for the other case could be explained by the general restrictions of PCR amplification from formalin-fixed and paraffin-embedded material or the involvement of other canine Mycoplasma species cross-reacting 
with the antisera used but not identified by selective species-specific PCR. It seems that M. cynos is not a frequent pathogen in CIRD and that also other $M y$ coplasma species, such as $M$. canis, may participate in this condition.

$\mathrm{CRCoV}$ is responsible for mild respiratory signs and is considered one of the aetiological agents of CIRD (Erles et al., 2004). The initial intention to use IHC for the identification of $\mathrm{CRCoV}$ in lung tissue was discarded after the available antibodies showed unspecific reactivity in control samples. So qRT-PCR was used instead. Due to the short amplification products generated by this procedure the negative effects of formalin fixation on the sensitivity of this method are considered less as compared to conventional PCRs producing larger amplicons. There were five cases in which recognisable amplification was generated; among them only one case had a $\mathrm{Cq}$ value compatible with a high viral load. The $\mathrm{Cq}$ values of the other four cases are associated with a low viral load and the clinical relevance of this finding is questionable (Spiss et al., 2012).

Finally, CAV-2 as another proven viral agent of CIRD is commonly isolated from more severe cases of kennel cough and is highly contagious. In the present study, using an ISH procedure which proved suitable for the detection of viral signals in tissue samples (Benetka et al., 2006; Chvala et al., 2007), no positive case was found. Thus, this virus does not seem to be a very common cofactor in canine pneumonias either.

Pneumocystis spp. is an opportunistic fungal pathogen of many different animal species as well as humans (Stringer, 1996), which can induce dyspnoea and pneumonia in dogs, especially in immunosuppressed individuals (Lobetti, 2012). In order to determine whether this agent was previously overlooked in the examined pneumonia cases a previously described ISH procedure was applied (Binanti et al., 2014). Pneumocystis spp. was not discovered in any of the cases which supports the assumption that Pneumocystis pneumonia is rare in dogs.

Although the chosen pathogens covered a range of well-known and established causative pathogens as well as more novel infectious agents which may lead to pneumonia, the number of positive results in the current investigation was moderate. However, the investigated pathogens covered only a part of possible CIRD-associated organisms. There are other viruses (e.g. canine herpesvirus, canine parainfluenza virus, canine reovirus, canine influenza virus, canine pneumovirus, pantropic canine coronavirus, canine bocavirus, and canine hepacivirus) and other bacteria (e.g. E. coli, Klebsiella pneumoniae, Streptococcus canis and Streptococcus equi subsp. zooepidemicus) which have been discussed to be part of the CIRD complex and which may also contribute to the development of respiratory disorders (Priestnall et al., 2014). These agents should be considered in similar studies in the future. 


\section{Acknowledgements}

The authors thank Ingrid Friedl, Karin Fragner and Karin Walk for their excellent technical assistance and Klaus Bittermann for his help with editing the digital images.

\section{References}

Benetka, V., Weissenböck, H., Kudielka, I., Pallan, C., Rothmüller, G. and Möstl, K. (2006): Canine adenovirus type 2 infection in four puppies with neurological signs. Vet. Rec. 158, 91-94.

Binanti, D., Mostegl, M. M., Weissenbacher-Lang, C., Nedorost, N. and Weissenböck, H. (2014): Detection of Pneumocystis infections by in situ hybridization in lung samples of Austrian pigs with interstitial pneumonia. Med. Mycol. 52, 196-201.

Caswell, J. L. and Williams, K. J. (2007): Respiratory system. In: Maxie, G. M. (ed.) Jubb, Kennedy and Palmer's Pathology of Domestic Animals, Volume 2. 5th edition, Elsevier Saunders, Edinburgh, New York. pp. 524-650.

Chalker, V. J. and Brownlie, J. (2004): Taxonomy of the canine Mollicutes by $16 \mathrm{~S}$ rRNA gene and $16 \mathrm{~S} / 23 \mathrm{~S}$ rRNA intergenic spacer region sequence comparison. Int. J. Syst. Evol. Micr. 54, $537-542$.

Chalker, V. J., Owen, W. M. A., Paterson, C., Barker, E., Brooks, H., Rycroft, A. N. and Brownlie, J. (2004): Mycoplasmas associated with canine infectious respiratory disease. Microbiol. 150, 3491-3497.

Chvala, S., Benetka, V., Möstl, K., Zeugswetter, F., Spergser, J. and Weissenböck, H. (2007): Simultaneous canine distemper virus, canine adenovirus type 2, and Mycoplasma cynos infection in a dog with pneumonia. Vet. Pathol. 44, 508-512.

Dear, J. D. (2014): Bacterial pneumonia in dogs and cats. Vet. Clin. North Am. Small Anim. Pract. 44, 143-159.

Erles, K., Dubovi, E. J., Brooks, H. W. and Brownlie, J. (2004): Longitudinal study of viruses associated with canine infectious respiratory disease. J. Clin. Microbiol. 42, 4524-4529.

Ford, R. B. (2012): Canine infectious respiratory disease. In: Greene, C. E. (ed.) Infectious Diseases of the Dog and Cat. 4th edition. Elsevier Saunders, St. Louis. pp. 55-65.

Lee-Fowler, T. and Reinero, C. (2012): Bacterial respiratory infections. In: Greene, C. E. (ed.) Infectious Diseases of the Dog and Cat. 4th edition. Elsevier Saunders, St. Louis. pp. 936-948.

Lobetti, R. (2012): Pneumocystosis. In: Greene, C. E. (ed.) Infectious Diseases of the Dog and Cat. 4th edition. Elsevier Saunders, St. Louis. pp. 689-696.

Priestnall, S. L., Mitchell, J. A., Walker, C. A., Erles, K. and Brownlie, J. (2014): New and emerging pathogens in canine infectious respiratory disease. Vet. Pathol. 51, 492-504.

Spiss, S., Benetka, V., Künzel, F., Sommerfeld-Stur, I., Walk, K., Latif, M. and Möstl, K. (2012): Enteric and respiratory coronavirus infections in Austrian dogs: serological and virological investigations of prevalence and clinical importance in respiratory and enteric disease. Vet. Med. Austria/Wien. Tierärztl. Mschr. 99, 67-81.

Stringer, J. R. (1996): Pneumocystis carinii: What is it, exactly? Clin. Microbiol. Rev. 9, 489-498. 